\title{
Neurological Manifestations of Patients Hospitalized with Coronavirus Disease 2019 in Babol, Iran
}

\author{
Ali Zabihi (iD) ${ }^{1}$, Seyedeh Roghayeh Jafarian Amiri (iD) ${ }^{2}$, Payam Saadat ${ }^{3}$, Hadi Ebrahimi (iD) ${ }^{4}$, Masoumeh \\ Bayani (iD ${ }^{5}$, Reyhaneh Babanataj ${ }^{6}$, Fatemeh Babaeian Keshteli ${ }^{7}$ and Mojtaba Qanbari Qalehsari (iD) ${ }^{2,}$ \\ ${ }^{1}$ Social Determinants of Health Research Center, Health Research Institute, Babol University of Medical Sciences, Babol, Iran \\ ${ }^{2}$ Nursing Care Research Center, Health Research Institute, Babol University of Medical Sciences, Babol, Iran \\ ${ }^{3}$ Department of Psychiatry, School of Medicine, Mobility Impairment Research Center, Research Institute for Health, Rouhani Hospital, Babol University of Medical Sciences, \\ Babol, Iran \\ ${ }^{4}$ Department of Surgery, School of Medicine, Rouhani Hospital, Babol University of Medical Sciences, Babol, Iran \\ ${ }^{5}$ Department of Infectious Disease, School of Medicine, Infectious Diseases and Tropical Medicine Research Center, Research Institute for Health, Rouhani Hospital, Babol \\ University of Medical Sciences, Babol, Iran \\ ${ }^{6}$ Department of Nursing, Babol University of Medical Sciences, Babol, Iran \\ ${ }^{7}$ Infectious Diseases and Tropical Medicine Research Center, Research Institute for Health, Rouhani Hospital, Babol University of Medical Sciences, Babol, Iran \\ "Corresponding author: Nursing Care Research Center, Health Research Institute, Babol University of Medical Sciences, Babol, Iran. Tel: +98-1132190597, Email: \\ m2ghanbari@yahoo.com
}

Received 2020 August 12; Revised 2020 September 29; Accepted 2020 September 29.

\begin{abstract}
Background: The coronavirus disease 2019 (COVID-19) has been associated with a wide range of clinical symptoms.

objectives: The present study aims to investigate the neurological manifestations among patients hospitalized with COVID-19 in Rouhani Hospital in Babol.

Methods: This is a descriptive cross-sectional survey conducted in March and April 2020 on patients admitted to Rouhani Hospital in Babol with COVID-19. A number of 230 medical records were selected randomly from a list. The data were collected using the demographic characteristics questionnaire and a checklist for the neurological symptoms.

Results: Among the 230 patients with COVID-19 (mean [SD] age, 59.85 [16.22] years; 138 men [60\%]), 127 (56\%) patients had at least one neurologic symptom. The most common symptoms of the central nervous system disorder were headache (34\%), dizziness (25\%), and impaired consciousness (20\%), and the most prevalent symptoms of the peripheral nervous system disorder were taste impairment (17\%) and smell impairment (16\%). The occurrence of transient ischemic attack (TIA) in women was significantly higher than in men $(\mathrm{P}=0.02)$. Moreover, the incidence of dizziness, impaired consciousness, ataxia, cerebrovascular accident $(\mathrm{CVA})$, anorexia, and smell impairment had a significant relationship with age $(\mathrm{P}<0.05)$.

Conclusions: The neurological manifestations are prevalent and substantial among patients with COVID-19. Therefore, it is suggested that the healthcare workers take these symptoms seriously, especially in the outbreak of COVID-19, and take the pertinent preventive and protective measures.
\end{abstract}

Keywords: Patients, Neurological Manifestations, Hospitalized, COVID-19

\section{Background}

Since the end of 2019, a new member of the coronavirus family, called severe acute respiratory syndromecoronavirus-2 (SARS-CoV-2), has been threatening the human society. The concernment regarding this new virus is because it is for the third time in less than two decades that the world faces a deadly epidemic caused by coronaviruses (1). Before 2002, it was thought that coronaviruses do not cause serious problems. Until for the first time, the outbreak of Severe Acute Respiratory Syndrome (SARS) in China and 29 other countries infected more than 8000 people and killed about 10 percent of the patients (2). In
2012, a new coronavirus affected the world for the second time. The disease caused by it was called Middle East Respiratory Syndrome (MERS) and originated in Saudi Arabia. Although it spread out more slowly than SARS, it infected 2494 people and killed 35 percent of them (3).

In December 2019, another coronavirus was discovered in Wuhan, China, the control of which has become a global concern these days. This virus causes serious and fatal respiratory problems (4), which are similar to the acute respiratory syndrome. It seems to infect elderly men more quickly than others (5). The importance and necessity of preventing the spread of COVID-19 lies in the fact that it is highly transmissible compared to SARS and MERS. More- 
over, its incubation period is long, and it takes 2 to 14 days for the symptoms to appear. This unique feature enables it to spread quickly and makes it harder to prevent and control the disease (4).

The typical symptoms of COVID-19 can range from mild to severe respiratory illness. The most common symptoms reported so far include fever, cough, and shortness of breath. Older adults, especially those with underlying medical conditions such as bronchitis, emphysema, heart failure, and diabetes, are at risk of developing the severe form of the disease (6). In a study in Wuhan, China, the most prevalent symptoms at the onset of the disease were: fever with 132 cases (61.7\%), cough with 107 cases (50\%), and anorexia with 68 cases (31.8\%). Moreover, 78 patients with COVID-19 (36.4\%) had nervous system manifestations that appeared in the form of central nervous system (CNS) disorders (8.24\%), peripheral nervous system (PNS) disorders (9.8\%), and musculoskeletal injuries (10.7\%). The most common symptoms of CNS disorders were dizziness (16.8\%) and headache (13.1\%), while the most common symptoms of PNS disorders were taste impairment (5.6\%) and smell impairment (5.1\%). The neurologic symptoms were significantly more prevalent in patients with more severe infection (45.5\% compared to $30.2 \%$ ). The most important neurological manifestations in these patients were acute cerebrovascular accidents (5.7\%), impaired consciousness (14.8\%), musculoskeletal injuries (19.3\%), and in one case, seizure symptoms (7).

The early symptoms of COVID-19 include fever, cough, and fatigue. But in areas affected with this disease, physicians found that some patients diagnosed with COVID-19 did not have the typical respiratory symptoms such as fever and cough at the time of diagnosis and only had the neurologic symptoms including headache, languidness, walking disability, and malaise. However, further investigations are required to confirm these symptoms as the non-specific and early symptoms of the disease (8) because they might be caused by cerebral hemorrhage (9), cerebral infarction (10), and other nerve diseases (11).

\section{Objectives}

Since Iran is one of the most highly affected by COVID19 in the world, this study aimed to investigate the neurological manifestations of patients hospitalized with COVID-19 in Rouhani Hospital in the city of Babol.

\section{Methods}

This descriptive cross-sectional study was conducted on patients hospitalized with COVID-19 in Rouhani Hospital, in Babol. It was conducted in March and April
2020, when the disease outbreak peaked, and only the patients from Babol and western cities of Mazandaran province, who had severe symptoms of COVID-19, were admitted to Rouhani Hospital. This study was approved by the research ethics committee of Babol University of Medical Sciences under the ethical approval code: IR.MUBABOL.REC.1399.183. In this study, the demographic information and the neurological manifestations of the hospitalized patients were extracted from their medical records using a checklist, and then the acquired data were analyzed. First, by presenting a letter of introduction from Babol University of Medical Sciences and after coordination with the CEO of the hospital, the researcher was granted access to the archives for the patients' medical records. The sampling process was as follows: the sampling method used in this study was convenience sampling. First, a list was made from the medical record numbers of all the patients who were infected with COVID-19 according to the results of their PCR tests. Then 230 medical records were randomly selected from 568 medical records of patients with COVID-19.

Before the approval of the present research project, a pilot study was conducted using 10 medical records to identify the potential problems of the project and also to revise the checklist. It showed that the majority of the records contained the information required for implementing this research project. After that, the main sampling was performed on 230 medical records based on a similar study (7). The inclusion criterion was being a hospitalized patient diagnosed with COVID-19, and the exclusion criterion was having an incomplete medical record.

The research tools used in this study were a demographic information questionnaire, which contained questions about age, gender, marital status, residence, occupation, etc., as well as a checklist for the neurologic manifestations, including the peripheral nervous system manifestations (such as taste, smell, and visual impairment, anorexia, fatigue, myalgia and other symptoms of cranial nerve involvement) and the central nervous system manifestations (including dizziness, headache, impaired consciousness, ataxia, CVA, TIA, encephalitis, cerebral hemorrhage, transverse myelitis, hydrocephaly, and neuralgia). After a detailed and comprehensive review of the patients' medical records, the researcher extracted the clinical manifestations documented in the records and put them in the checklist. The criterion for the presence or absence of the neurologic symptoms was the researcher's study of the medical record, which was reflected through the pertinent option in the researcher-made checklist (in the form of a YES/NO question). This checklist was finalized after it was presented to 10 clinical experts to ask for their opinions and use them in the present study. The data, which 
were collected and kept anonymously and confidentially, were entered in the SPSS18 software and analyzed using chisquare and t-test. Moreover, a p-value of less than 0.05 was considered to be significant.

\section{Results}

In this study, 230 medical records of patients with COVID-19 were investigated. The patients' ages ranged from 16 to 97 years (mean $=59.85, \mathrm{SD}=16.22$ ) with $60 \%$ identified as male, $30 \%$ reported being self-employed or freelancer, 51\% had less than grade 12 (high school) education, and $58 \%$ resided in the city, and the rest of them lived in rural areas. Only 15\% of hospitalized patients were under 40 years of age. The most prevalent typical symptoms among the patients hospitalized with COVID19 included fever (87\%), respiratory distress (87\%), cough (86.1\%), fatigue (77.8\%), myalgia (73\%), and anorexia (48\%) and the most common neurological manifestations were headache (34\%), dizziness (25\%), impaired consciousness (20\%), taste impairment (17\%), and smell impairment (16\%). Of these patients, 127 (56\%) had at least 1 of the neurological manifestations (According According to Table 1, the rate of TIA is significantly more in female compared to male patients $(\mathrm{P}=0.02)$.

Apart from the typical symptoms such as fever, cough, and shortness of breath, other symptoms observed among these patients included nausea (1\%), chills (2.6\%), earache $(1 \%)$, and diarrhea (3.4\%).

Among the typical symptoms, anorexia was significantly more prevalent in the age groups of under 40 and over 60 compared to the age group of 40 to $60(\mathrm{P}=0.03)$. Respiratory distress was observed to be more common in the age group of over 40 compared to the age group of under $40(\mathrm{P}=0.02)$. There were statistically significant differences in the general symptoms of the nervous system and the central nervous system, including dizziness $(\mathrm{P}=$ $0.008)$, impaired consciousness $(\mathrm{P}=0.02)$, ataxia $(\mathrm{P}=0.04)$, CVA $(\mathrm{P}=0.01)$ in different age groups (Table 2 ).

\section{Discussion}

The results of the present study indicated a high and considerable prevalence of the typical symptoms (fever, cough, and respiratory distress) in patients with COVID19. In this study, $56 \%$ of the hospitalized patients had neurological manifestations, including headache (34\%), dizziness (25\%), impaired consciousness (20\%), taste impairment (17\%), smell impairment (16\%), and CVA (4.3\%). In a similar study in Wuhan, China $(n=214), 45.5 \%$ of the patients with severe infection had neurologic symptoms of which the most common ones were dizziness (19.3\%), headache (17\%), impaired consciousness (14.8\%), taste impairment (3.4\%), smell impairment (3.14\%), and acute cerebrovascular disease (5.7\%) (7). The comparison of the results of the two studies reveals that the prevalence of the neurologic symptoms is higher in the present study compared to the one in Wuhan, China.

The prevalence of the typical symptoms was higher in the present study compared to the study in Wuhan. While the prevalence of fever and cough in the present study was respectively $87 \%$ and $86.1 \%$, the prevalence of these items in the study in Wuhan was $45.5 \%$ and $34.1 \%$ (7). In another study in Wuhan, $80.3 \%$ of the patients had fever and $34.6 \%$ had cough (3). In the present study, $77.8 \%$ of the patients experienced fatigue, $73 \%$ of them had myalgia, and $48.3 \%$ of them had anorexia, while in a study in Wuhan, which investigated cardiac injury and mortality in patients with COVID-19, fatigue (13.2\%), myalgia (4.6\%), headache $(2.2 \%)$ and cerebrovascular diseases (5.3\%) were reported (12). The difference in the prevalence of the symptoms of the disease in the two studies might be due to the change in the virus genome caused by the passage of time and the genetic differences in the patients.

As shown in Table 1, there is no significant difference between the two genders in terms of the general and the neurologic symptoms. Although the number of CVA cases was higher in women than in men, the difference was not statistically significant. However, the TIA cases were only observed in women, and the difference in this regard was statistically significant. These findings have not been inspected in the previous studies. The occurrence of CVA and TIA in patients with COVID-19 is probably caused by the incidence of vasculitis that follows this disease. Therefore, the higher prevalence of CVA and TIA cases in women compared to men can be suggestive of the idea that, COVID-19 causes vasculitis more in women than in men.

According to Table 2, some clinical characteristics were statistically different among the age groups. For instance, the CNS symptoms were more prevalent in the age groups under 40 and over 60 compared to the middle-aged group. The CNS involvement might be caused by the virus reaching this area through the hematogenous or the retrograde neuronal route. In addition, headache was more common in the age groups under 40 and over 60 compared to the age group of 40-60, though this difference was not statistically significant. On the other hand, the prevalence of dizziness in the age groups under 40 and over 60 was significantly higher than the age group of 40-60. This discrepancy might be associated with the different behavior of the virus in different age groups considering their physiological status. The similar studies did not compare the incidence of headache and dizziness in different age groups. 


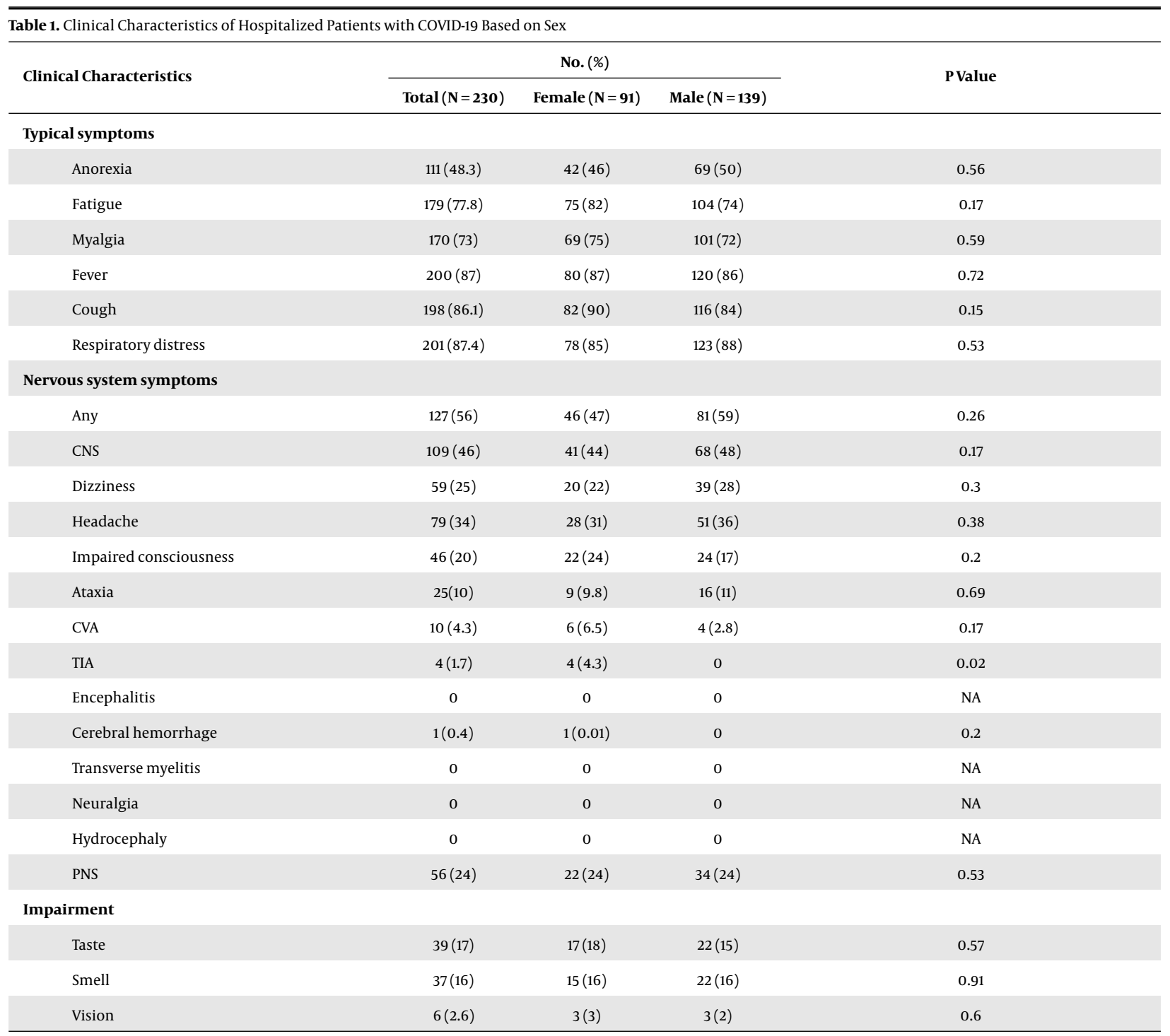

Abbreviation: NA, not applicable.

As Table 2 shows, the impaired consciousness increases significantly with the patients' age, in a way that a notable increase can be seen among the elderly patients, which can be due to chronic diseases and the aging process. In other studies, too, it was shown that the prevalence of impaired consciousness was higher in the hospitalized elderly patients than in other age groups $(13,14)$. The CVA cases were only seen among the patients over 60 years of age and were not reported in younger age groups. In the study of Coco et al., the relationship between the prevalence of stroke and old age was shown (14).

The prevalence of ataxia was significantly lower in the age group of 40-60 compared to other age groups, especially the elderly ones. In another study, it was shown that the prevalence of ataxia increased with age (15). In the present study, the lower prevalence of ataxia in the age group of 40-60 requires further studies in this regard.

Almost half of the patients with COVID-19 in the present study experienced anorexia, and it was significantly more prevalent in the age groups under 40 and over 60. In general, anorexia is a common symptom of many acute infectious diseases (16). It requires further investigations to find the reason why anorexia was less prevalent in middle-aged patients compared to the other age groups. Taste impairment was more common in younger patients, and the prevalence decreased with age, but the difference was not meaningful. However, the incidence rate of smell disorder was significantly higher in younger patients com- 


\begin{tabular}{|c|c|c|c|c|}
\hline \multirow{2}{*}{ Clinical Characteristics } & \multicolumn{3}{|c|}{ No. (\%) } & \multirow{2}{*}{ P Value } \\
\hline & $<40(\mathrm{~N}=35)$ & $40-60(N=73)$ & $>60(\mathrm{~N}=122)$ & \\
\hline \multicolumn{5}{|l|}{ Typical symptoms } \\
\hline Anorexia & $17(48)$ & $26(36)$ & $68(55)$ & 0.03 \\
\hline Fatigue & $25(71)$ & $55(75)$ & $99(81)$ & 0.39 \\
\hline Myalgia & $26(74)$ & $54(73)$ & $90(73)$ & 0.99 \\
\hline Fever & $31(88)$ & $67(91)$ & $102(83)$ & 0.24 \\
\hline Cough & $30(85)$ & $67(82)$ & $101(82)$ & 0.21 \\
\hline Respiratory distress & $26(74)$ & $68(93)$ & $107(87)$ & 0.02 \\
\hline \multicolumn{5}{|l|}{ Nervous system symptoms } \\
\hline Any & $20(57)$ & $30(42)$ & $77(63)$ & 0.019 \\
\hline $\mathrm{CNS}$ & $16(45)$ & $19(26)$ & $70(57)$ & 0.02 \\
\hline Dizziness & $10(28)$ & $9(12)$ & $40(32)$ & 0.008 \\
\hline Headache & $15(42)$ & $17(23)$ & $47(38)$ & 0.057 \\
\hline Impaired consciousness & $3(8)$ & $8(10)$ & $35(28)$ & 0.02 \\
\hline Ataxia & $3(8)$ & $3(4)$ & $19(15)$ & 0.04 \\
\hline CVA & 0 & 0 & $10(8)$ & 0.01 \\
\hline TIA & 0 & $1(1)$ & $3(2)$ & 0.59 \\
\hline Encephalitis & 0 & 0 & 0 & NA \\
\hline Cerebral hemorrhage & 0 & 0 & $1(0.8)$ & 0.64 \\
\hline Transverse myelitis & 0 & 0 & 0 & NA \\
\hline Neuralgia & 0 & 0 & 0 & NA \\
\hline Hydrocephaly & 0 & 0 & 0 & NA \\
\hline PNS & $12(34)$ & $20(28)$ & $23(18)$ & 0.4 \\
\hline \multicolumn{5}{|l|}{ Impairment } \\
\hline Taste & $10(28)$ & $13(17)$ & $16(13)$ & 0.09 \\
\hline Smell & $9(27)$ & $15(20)$ & $13(10)$ & 0.04 \\
\hline Vision & $1(3)$ & $1(1.5)$ & $4(3)$ & 0.72 \\
\hline
\end{tabular}

Abbreviation: NA, not applicable.

pared to the older ones. In the study of Lechien et al., the prevalence of smell and taste impairment was respectively $85 \%$ and $88 \%$ in patients with the mild to moderate form of COVID-19 (17). The study of Mao et al. showed that elderly people develop more severe forms of this disease (7).

There was no evidence of encephalitis or transverse myelitis in the present study. Other similar studies have not reported these complications $(7,12)$. In the study of Filatov, the analysis of the cerebrospinal fluid (CSF) of COVID19 patients showed no trace of coronavirus. It seemed, COVID-19 probably cannot cross the blood-brain barrier (18). However, other studies have reported these complications $(19,20)$.

The rest of the clinical characteristics did not have any significant relationship with the age groups.

One of the strengths of the present study was that the medical records of the hospitalized patients were carefully reviewed. Moreover, the city of Babol in north of Iran was among the cities with a considerably high prevalence of COVID-19. At that time (peak outbreak of the disease), Rouhani Hospital in Babol only admitted COVID-19 patients from the nearby region and the western cities of the Mazandaran province. The limitation of this study was that some of the medical records were incomplete.

\subsection{Conclusions}

Based on the findings of the present study, the neurological manifestations, including headache, dizziness, 
taste impairment, and smell impairment, were substantially prevalent in COVID-19 patients. Some of the neurologic symptoms were more common in different age groups. For instance, the cases of CVA and TIA were more common in women than in men. Therefore, the medical staff should take these symptoms seriously and consider the patients' age and gender in emergency centers and healthcare clinics to adopt appropriate preventive and therapeutic measures in time.

\section{Acknowledgments}

We would like to express our gratitude to the vicechancellor for the research and technology of Babol University of Medical Sciences for providing the financial support for this research project (Approved project code: 9909712). We would also like to thank the medical staff, especially the nurses, at Rouhani Hospital in Babol, who helped us with completing and reviewing the patients' medical records.

\section{Footnotes}

Authors' Contribution: AZ and MQQ conceived the idea of the study; RB and FB acquired the data; MQQ carried out the statistical analysis; PS, HE, and MB interpreted the findings; and AZ drafted the manuscript. MQQ had full access to all the data in the study and takes responsibility for the integrity of the data and the accuracy of the data analysis. MQQ, AZ and PS provided input in the analysis; all authors critically reviewed the manuscript and MQQ revised the manuscript for final submission.

Conflict of Interests: No conflict of interest to declare.

Ethical Approval: IR.MUBABOL.REC.1399.183.

Funding/Support: This work was supported by the vicechancellor for research and technology of Babol University of Medical Sciences.

\section{References}

1. Wilson ME, Chen LH. Travellers give wings to novel coronavirus (2019nCoV). J Travel Med. 2020;27(2). doi: 10.1093/jtm/taaa015. [PubMed: 32010938]. [PubMed Central: PMC7107561].

2. Zhu N, Zhang D, Wang W, Li X, Yang B, Song J, et al. A Novel Coronavirus from Patients with Pneumonia in China, 2019. N Engl J Med. 2020;382(8):727-33. doi: 10.1056/NEJMoa2001017. [PubMed: 31978945]. [PubMed Central: PMC7092803].

3. Zhang L, Lin D, Kusov Y, Nian Y, Ma Q, Wang J, et al. alpha-Ketoamides as Broad-Spectrum Inhibitors of Coronavirus and Enterovirus Replication: Structure-Based Design, Synthesis, and Activity Assessment. J Med Chem. 2020;63(9):4562-78. doi: 10.1021/acs.jmedchem.9b01828. [PubMed: 32045235]. [PubMed Central: PMC7098070].

4. Gralinski LE, Menachery VD. Return of the Coronavirus: 2019nCoV. Viruses. 2020;12(2). doi: 10.3390/v12020135. [PubMed: 31991541]. [PubMed Central: PMC7077245].
5. Chen N, Zhou M, Dong X, Qu J, Gong F, Han Y, et al. Epidemiological and clinical characteristics of 99 cases of 2019 novel coronavirus pneumonia in Wuhan, China: a descriptive study. Lancet. 2020;395(10223):507-13. doi:10.1016/s0140-6736(20)30211-7.

6. Wang D, Hu B, Hu C, Zhu F, Liu X, Zhang J, et al. Clinical Characteristics of 138 Hospitalized Patients With 2019 Novel CoronavirusInfected Pneumonia in Wuhan, China. JAMA. 2020;323(11):1061-9. doi: 10.1001/jama.2020.1585. [PubMed: 32031570]. [PubMed Central: PMC7042881].

7. Mao L, Jin H, Wang M, Hu Y, Chen S, He Q, et al. Neurologic Manifestations of Hospitalized Patients With Coronavirus Disease 2019 in Wuhan, China. JAMA Neurol. 2020;77(6):683-90. doi: 10.1001/jamaneurol.2020.1127. [PubMed: 32275288]. [PubMed Central: PMC7149362].

8. Li Q, Guan X, Wu P, Wang X, Zhou L, Tong Y, et al. Early Transmission Dynamics in Wuhan, China, of Novel Coronavirus-Infected Pneumonia. N Engl J Med. 2020;382(13):1199-207. doi: 10.1056/NEJMoa2001316. [PubMed: 31995857]. [PubMed Central: PMC7121484].

9. Huang C, Wang Y, Li X, Ren L, Zhao J, Hu Y, et al. Clinical features of patients infected with 2019 novel coronavirus in Wuhan, China. Lancet. 2020;395(10223):497-506. doi: 10.1016/S0140-6736(20)30183-5. [PubMed: 31986264]. [PubMed Central: PMC7159299].

10. Mao L, Wang M, Chen S; et al. Neurological manifestations of hospitalized patients with COVID-19 in Wuhan, China: a retrospective case series study. medRxiv. 2020. doi: 10.1101/2020.02.22.20026500.

11. Xu X, Chen P, Wang J, Feng J, Zhou H, Li X, et al. Evolution of the novel coronavirus from the ongoing Wuhan outbreak and modeling of its spike protein for risk of human transmission. Sci China Life Sci. 2020;63(3):457-60. doi: 10.1007/s11427-020-1637-5. [PubMed: 32009228]. [PubMed Central: PMC7089049].

12. Shi S, Qin M, Shen B, Cai Y, Liu T, Yang F, et al. Association of Cardiac Injury With Mortality in Hospitalized Patients With COVID-19 in Wuhan, China. JAMA Cardiol. 2020;5(7):802-10. doi: 10.1001/jamacardio.2020.0950. [PubMed: 32211816]. [PubMed Central: PMC7097841].

13. Andreas S, Schulz H, Volkert J, Dehoust M, Sehner S, Suling A, et al. Prevalence of mental disorders in elderly people: the European MentDis_ICF65+ study. Br J Psychiatry. 2017;210(2):125-31. doi: 10.1192/bjp.bp.115.180463. [PubMed: 27609811].

14. Lo Coco D, Lopez G, Corrao S. Cognitive impairment and stroke in elderly patients. Vasc Health Risk Manag. 2016;12:105-16. doi: 10.2147/VHRM.S75306. [PubMed: 27069366]. [PubMed Central: PMC4818041].

15. Kashyap R, Sharma S, Singh TP, Vishnoi V, Thakur MK; Laxaminand. Clinical profile of neurological gait ataxia: a hospital based study. Int J Res Med Sci. 2017;5(3):782. doi:10.18203/2320-6012.ijrms20170437.

16. Maki N, Nakatani E, Ojima T, Nagashima T, Harada T, Koike F, et al. The cause of anorexia and proportion of its recovery in older adults without underlying disease: Results of a retrospective study. PLoS One. 2019;14(10). e0224354. doi: 10.1371/journal.pone.0224354. [PubMed: 31648285]. [PubMed Central: PMC6812872].

17. Lechien JR, Chiesa-Estomba CM, De Siati DR, Horoi M, Le Bon SD, Rodriguez A, et al. Olfactory and gustatory dysfunctions as a clinical presentation of mild-to-moderate forms of the coronavirus disease (COVID-19): a multicenter European study. Eur Arch Otorhinolaryngol. 2020;277(8):2251-61. doi: 10.1007/s00405-020-05965-1.

18. Filatov A, Sharma P, Hindi F, Espinosa PS. Neurological Complications of Coronavirus Disease (COVID-19): Encephalopathy. Cureus. 2020. doi: $10.7759 /$ cureus.7352.

19. Chow CCN, Magnussen J, Ip J, Su Y. Acute transverse myelitis in COVID19 infection. BMJ Case Rep. 2020;13(8). doi: 10.1136/bcr-2020-236720. [PubMed: 32784242]. [PubMed Central: PMC7418849].

20. Chakraborty U, Chandra A, Ray AK, Biswas P. COVID-19-associated acute transverse myelitis: a rare entity. BMJ Case Rep. 2020;13(8). doi: 10.1136/bcr-2020-238668. [PubMed: 32843475]. [PubMed Central: PMC7449353]. 